CONTRIBUCIÓN DE LA TÉCNICA DEL GRUPO FOCAL AL ACERCAMIENTO A LA PERCEPCIÓN ESTUDIANTIL SOBRE ACCESIBILIDAD EN EL ENTORNO UNIVERSITARIO

TECHNICAL CONTRIBUTION TO FOCUS GROUP APPROACH TO STUDENTS PERCEPTION ON ACCESSIBILITY IN UNIVERSITY ENVIRONMENT

\author{
Volumen 15, Número 1 \\ Enero - Abril \\ pp. 1-16
}

Este número se publicó el $1^{\circ}$ de enero de 2015

DOI: dx.doi.org/10.15517/aie.v15i1.17587

Martha Gross Martínez

Laura Stiller González

Revista indizada en REDALYC, $\underline{\text { SCIELO }}$

Revista distribuida en las bases de datos:

CATÁLOGO DE LATINDEX, IRESIE, CLASE, DIALNET, DOAJ, E-REVIST@S, SHERPA/ROMEO, QUALIS, MIAR

Revista registrada en los directorios:

ULRICH'S, REDIE, RINACE, OEI, MAESTROTECA, PREAL, CLACSO 


\title{
CONTRIBUCIÓN DE LA TÉCNICA DEL GRUPO FOCAL AL ACERCAMIENTO A LA PERCEPCIÓN ESTUDIANTIL SOBRE ACCESIBILIDAD EN EL ENTORNO UNIVERSITARIO \\ TECHNICAL CONTRIBUTION TO FOCUS GROUP APPROACH TO STUDENTS PERCEPTION ON ACCESSIBILITY IN UNIVERSITY ENVIRONMENT
}

\author{
Martha Gross Martínez ${ }^{1}$ \\ Laura Stiller González
}

\begin{abstract}
Resumen: Este artículo describe la técnica de grupo focal utilizada en la investigación: "Accesibilidad en la permanencia de estudiantes con necesidades educativas asociadas o no a discapacidad: Sede Rodrigo Facio" de la Universidad de Costa Rica; cuyo objetivo general consistió en la elaboración de una guía de comprobación y buenas prácticas en el tema de accesibilidad, como un recurso que le permita a la comunidad universitaria dar respuesta a esta población y constituirse en insumo para la construcción de una universidad inclusiva. A partir de los grupos focales, se exploró la percepción estudiantil con necesidades educativas asociadas o no a discapacidad, sobre la accesibilidad en la vida universitaria; para ello esta se caracterizó y categorizó en tres dimensiones: accesibilidad al espacio físico, a la información y al proceso educativo. Esta técnica permitió que cada participante expresara sus percepciones, comentara su vivencia e interacción con el entorno universitario. Finalmente, se concluye que los grupos focales constituyen una técnica relevante de recolección de información en los procesos de investigación cualitativa, porque establecen un acercamiento personal, lo que la convierte en un recurso útil para conocer a esta población.
\end{abstract}

Palabras clave: ACCESIBILIDAD, UNIVERSIDAD INCLUSIVA, PERCEPCIÓN, GRUPOS FOCALES, UNIVERSIDAD DE COSTA RICA

\begin{abstract}
This article describes the focal group technique employed during the research: Accessibility in the Permanence of Students with Special Educational Needs Associated or not to a Disability: Rodrigo Facio Headquarters. The general objective was to elaborate good practice and verification guidelines on the area of accessibility. This resource would permit the university community to come close to this population; it would also provide a tool to help build an inclusive university. The focal groups helped explore the perception of students about accessibility in their university life. This was characterized and categorized in three dimensions: accessibility in physical spaces, accessibility to information, and accessibility to the educational process. The dynamic of this technique expresses the different opinions of the students related to the aspects that have helped them move forward with their university life, as well as those experiences that have been negative and that have represented relevant obstacles in the context of their educational process. It also helped students express their perceptions, and talk about their experiences and interactions on the university campus. The conclusion is that focal groups constitute a relevant information compilation technique in the processes of qualitative research, since it establishes a persona approach with the participants of the research process
\end{abstract}

Key Words: ACCESSIBILITY, INCLUSIVE UNIVERSITY, PERCEPTION, FOCAL GROUPS, UNIVERSITY OF COSTA RICA

\footnotetext{
${ }^{1}$ Docente de la carrera de Educación Especial, Universidad de Costa Rica. Máster en Psicopedagogía y Licenciada en Educación Especial. Dirección electrónica: martha.gross@ucr.ac.cr

2 Psicopedagoga en el Centro de Asesoría y Servicios al Estudiante con discapacidad, de la Universidad de Costa Rica, representante en la CISO. Doctora en Educación con énfasis en mediación pedagógica, Maestría en Psicopedagogía y Licenciatura en Psicología. Dirección electrónica: laura.stiller@ucr.ac.cr
}

Artículo recibido:4 de marzo, 2014

Devuelto a corrección: 21 de julio, 2014

Aprobado: $1^{\circ}$ de diciembre, 2014 


\section{Introducción}

La población estudiantil con necesidades educativas asociada o no a discapacidad, que accede a la educación superior va en continuo crecimiento, como resultado de las políticas y legislación inclusivas (nacional e internacional) sobre el derecho a la educación. Esto ha hecho necesario, realizar ajustes en concordancia con los requerimientos generales y específicos del quehacer en las aulas universitarias.

La Universidad de Costa Rica ha sido una institución de educación superior pionera en acciones vinculadas a la accesibilidad: ha logrado sensibilizar, concienciar a la comunidad universitaria y enseñar que la diferencia entre cada persona es lo que hace que se trabaje por el respeto hacia ellas y se logre un sistema social y educativo más justo para toda la población universitaria: "Dentro de la igualdad se instaura la diferencia, entendida como apertura del uno al otro, es decir, como reciprocidad" (Boff y Muraro, 2002, p. 81).

Las más recientes políticas inclusivas, como la Declaración de los Derechos de las Personas con Discapacidad, ratificada en Costa Rica como la Ley 8661, fortalece las acciones que permiten la inclusión de esta población en igualdad de oportunidades. Cabe destacar que la Universidad de Costa Rica ha brindado asesoría tanto a nivel nacional como en el ámbito regional, siempre en una línea de visión de los derechos e igualdad de oportunidades, bajo un modelo social (Stupp, 2005).

En este contexto y como respuesta a la necesidad de indagar sobre la accesibilidad en la Sede Rodrigo Facio, se planteó la siguiente investigación: "Accesibilidad en la permanencia de estudiantes con necesidades educativas asociadas o no a discapacidad: Sede Rodrigo Facio". El objetivo general consistió en la elaboración de una guía de información y servicios en el tema de accesibilidad, como un recurso que le permita a la comunidad universitaria dar respuesta a esta población.

Además, se indagó la percepción de la población estudiantil con necesidades educativas asociadas o no a discapacidad, sobre la accesibilidad en la vida estudiantil universitaria. Con este propósito se caracterizó y categorizó la accesibilidad en tres dimensiones: accesibilidad al espacio físico, accesibilidad a la información y accesibilidad al proceso educativo.

A continuación se detallará sobre la accesibilidad en el entorno universitario, se conceptualizan las dimensiones de la accesibilidad y la percepción del estudiantado explorada por medio de la técnica de grupos focales. 


\section{Accesibilidad en el entorno universitario}

La investigación: "Accesibilidad en la permanencia de estudiantes con necesidades educativas asociadas o no a discapacidad: Sede Rodrigo Facio", surge a partir de las inquietudes generadas con respecto a la población estudiantil con necesidades educativas asociadas o no a discapacidad, adscrita al Artículo 37 del Reglamento de régimen académico estudiantil y del análisis sobre el proceso de permanencia de esta población: Consecuentemente se encontró un vacío en el área de investigación y en la sistematización de los apoyos y requerimientos en la vida estudiantil en cuanto a la accesibilidad al espacio físico, a la información y al proceso educativo.

La Universidad de Costa Rica, a través del Centro de asesoría y servicios al estudiante con discapacidad (CASED), la Escuela de Orientación y Educación Especial y otras instancias universitarias, han hecho esfuerzos significativos, por fortalecer los servicios y estrategias de apoyo que dan el asesoramiento y soporte para hacer efectiva una universidad inclusiva, con alternativas y propuestas acorde a los requerimientos de la población estudiantil con necesidades educativas, asociada o no a discapacidad.

Cuando se trata el tema de la accesibilidad se toma en cuenta la normativa vigente a nivel institucional, así como la legislación en la temática a nivel nacional, comprendidas ambas dentro de la Ley 8661, Convención de los Derechos de las Personas con Discapacidad y la Ley 7600 de Igualdad de Oportunidades, en estas se define la accesibilidad como la "propiedad que tienen algunos lugares a los que se puede llegar o entrar fácilmente. También es la propiedad que tienen algunas cosas que se comprenden con facilidad" (Costa Rica, Asamblea Legislativa, 2010, p. 14).

Como resultado de la revisión teórica que fundamenta la investigación se definieron la categoría accesibilidad y las subcategorías correspondientes (espacio físico, información y proceso educativo), con el fin de precisar la percepción de la accesibilidad por parte de los estudiantes con necesidades educativas, asociadas o no a discapacidad, adscritos al Artículo 37 del Reglamento de Régimen Académico Estudiantil. De esta forma se presentan a continuación la conceptualización de las subcategorías:

- Accesibilidad, se entiende como el derecho que tiene la persona para hacer uso efectivo del entorno físico, de los recursos impresos, visuales y audibles y del currículum universitario, de forma segura y autónoma.

- Acceso al espacio físico: se refiere a la posibilidad de llegar, hacer uso e interactuar a un lugar o entorno, de forma autónoma e independiente. 
- Acceso a la información, está relacionado con la oportunidad de poder utilizar tanto el material impreso, audible y digital, en las diferentes actividades de la vida universitaria: por ejemplo, los trámites administrativos, recreativos y de tipo académico.

- Acceso al proceso educativo, comprende la disposición de los recursos necesarios para que la estudiante o el estudiante participen en igualdad de condiciones en el proceso de aprendizaje.

Los aspectos descritos anteriormente, comprenden elementos fundamentales para la inclusión educativa en el ámbito universitario, estos garantizan tanto el acceso como la participación de la población estudiantil adscrita al Artículo 37 del Régimen Académico Estudiantil.

\section{Percepción estudiantil}

En general, la percepción se ha concebido como un proceso cognoscitivo para obtener información a través de los sentidos; construyendo una imagen de la realidad; este procesamiento involucra además los conocimientos y experiencias previas.

Las personas manifiestan sus percepciones según su contexto social y cultural, esto supone procesos de interacción y comunicación. Al respecto Abbagnano citada por Vargas (2013) define la percepción de la siguiente manera:

La percepción no es un proceso lineal de estímulo y respuesta sobre un sujeto pasivo, sino que, por el contrario, están de por medio una serie de procesos en constante interacción y donde el individuo y la sociedad tienen un papel activo en la conformación de percepciones particulares a cada grupo social. En el proceso de la percepción están involucrados mecanismos vivenciales que implican tanto al ámbito consciente como al inconsciente de la psique humana (p. 48).

Para esta investigación ha sido fundamental, conocer la percepción sobre accesibilidad en la Sede Rodrigo Facio de las y los estudiantes adscritos al artículo 37 del Reglamento de Régimen Académico Estudiantil; por cuanto ellas y ellos son protagonistas del quehacer universitario: tanto en la docencia como en la vida estudiantil.

La vida estudiantil universitaria, enfocada desde una visión humanística y una perspectiva de atención integral, contempla acciones, programas y servicios para el desarrollo personal y social de la población estudiantil, con la cual brinda las condiciones necesarias para el "mejoramiento del educando como individuo, como universitario y como 
miembro solidario de la sociedad" (Universidad de Costa Rica, Vicerrectoría de Vida Estudiantil, 2013, artículo 1).

La Vicerrectoría de Vida Estudiantil promueve la igualdad de oportunidades en el ámbito universitario para toda la población estudiantil; desarrollando y apoyando acciones que incentivan la inclusión a la vida universitaria de las y los estudiantes con discapacidad (Universidad de Costa Rica, Vicerrectoría de Vida Estudiantil, 2013).

La Universidad de Costa Rica, en su Estatuto Orgánico establece los procesos de admisión, permanencia y graduación, desde el quehacer del Área de Vida Estudiantil, por medio del conjunto de programas y servicios que se desarrollan en cada uno de estos procesos:

- Admisión: conjunto de acciones enmarcadas dentro del proceso de ingreso del estudiante a la Universidad de Costa Rica y sus diferentes etapas, a saber: la inscripción y la aplicación a la prueba de Aptitud Académica; así como el concurso y la aceptación del estudiante en una carrera.

- Permanencia: conjunto de acciones que contribuyen a la consolidación del ingreso a carrera (matrícula) por parte del estudiantado y a la continuación satisfactoria hasta la conclusión del plan de estudio.

- Graduación: conjunto de acciones que se dirigen a las y los estudiantes quienes se encuentran en el último periodo del plan de estudios hasta la obtención de un título universitario. Se plantean, en esta etapa, relaciones con posgrado, becas de estudio y proceso laboral.

Para detallar todos los aspectos que contempla la vida estudiantil en la Sede Rodrigo Facio, entre los que se consideran las diferentes actividades de la vida universitaria, como trámites administrativos, recreativos y de tipo académico, según la categoría de accesibilidad correspondiente. Se elaboró un mapa (anexo) que permite visualizar la cotidianeidad de la universitaria y el universitario, mediante la identificación de los diferentes procesos de manera integrada y el ligamen con las subcategorías de la accesibilidad, estas son el acceso al espacio, el acceso a la información y por último el acceso al proceso educativo.

\section{Técnica de grupo focal}

Para la recopilación de información de nuestra investigación se seleccionó, la técnica de grupo focal ya que por sus características y propósitos posibilita información precisa y 
relevante para el tema de estudio. En este sentido, Huerta (2014) define esta técnica de la siguiente manera:

El grupo focal es una herramienta muy útil para la planificación de los programas y la evaluación de los mismos. El secreto consiste en que los participantes puedan expresar libremente su opinión sobre diferentes aspectos de interés en un ambiente abierto para el libre intercambio de ideas. Otro de los aspectos positivos estriba en el hecho de proveer participación a las personas involucradas en los respectivos programas. (p. 1)

La técnica del grupo focal permitió que cada participante expresara sus percepciones y comentara su vivencia e interacción en el entorno universitario, desde las tres dimensiones de la investigación, a saber proceso educativo, información y espacio físico. En el intercambio, se expresaron las diferentes opiniones de las y los estudiantes en relación con los aspectos que les han permitido avanzar en su vida universitaria, así como en las experiencias negativas que han representado barreras en el contexto de su proyecto educativo.

En este sentido Korman citado por Lema y Agila (2008), en su conceptualización de grupo focal explica que esta técnica constituye "Una reunión de un grupo de individuos seleccionados por los investigadores para discutir y elaborar, desde la experiencia personal, una temática o hecho social que es objeto de investigación" (p. 10).

Así los grupos focales son de gran utilidad para obtener información de aspectos relacionados con temas de investigación, al tomar en cuenta la experiencia directa de los involucrados.

Sandoval (2002) explica que en la planificación de un grupo focal se deben de llevar a cabo algunas pautas que guíen el proceso de aplicación, esto incide en la calidad de la recolección de los insumos aportados por las y los participantes ofreciendo algunos lineamientos para su ejecución, a saber:

1- Cantidad de grupos que se llevarán a cabo para la obtención de la información:

en la presente investigación se determinó que a partir de la respuesta de convocatoria (vía correo electrónico y por llamadas telefónicas) de las y los estudiantes, se ofrecerían varias opciones de horarios, en los que se contemplaría la variedad de agendas y compromisos de las y los participantes. En este sentido se abrió la oferta de 
7 opciones de horario, sin embargo sólo se logró abrir tres grupos (uno por la mañana y dos por la tarde).

2- Tamaño de los grupos, el rango varía de 6-8 participantes como mínimo:

en relación con este aspecto la configuración de los grupos de la investigación resultó de la siguiente forma: al primer grupo asistieron 9 estudiantes, al segundo fueron 6 estudiantes y al último grupo acudieron 4 estudiantes.

\section{3- Roles de las personas investigadoras:}

este aspecto constituyó un elemento relevante para la dinámica de los grupos focales; se contó con la figura de la Facilitadora quien fue la responsable de dirigir la agenda de trabajo y motivar al grupo a una participación asertiva, ella guió a través preguntas vinculadas con los componentes de la investigación que generaron los comentarios. Además, les explicó el objetivo de la actividad, les detalló la metodología de trabajo en el grupo focal y les solicitó su autorización para grabar los comentarios.

El otro rol fue la figura de la Colaboradora, quien tomó apuntes de las opiniones y de la dinámica presentada, confeccionó una síntesis de las sesiones y aunado a ello realizó el cierre de las ideas y comentarios expuestos durante la actividad.

\section{Percepción estudiantil sobre accesibilidad}

A continuación se presentan los resultados obtenidos en los grupos focales desde las tres dimensiones de la investigación:

\section{Accesibilidad al espacio físico}

Entre los principales hallazgos de esta dimensión se percibe que la mayoría de las y los participantes del grupo focal, manifestaron que aún cuando hay una tendencia a la construcción de rampas en la mayoría de los edificios del campus universitario, éstas se hallan mal construidas y no tienen las medidas o características idóneas. Al respecto un estudiante comenta: "La salida trasera del edificio de Farmacia, hacia el CASE, no posee accesibilidad, es un camino que no cuenta con rampas y son solamente piedras".

En cuanto a la iluminación, expresan que se han hecho esfuerzos en tener una Sede con iluminación adecuada y perciben que se están haciendo trabajos por mejorar la calidad de esta:

“De Ciencias Sociales a Ciencias Económicas sí hay buena iluminación, pero no todos los días de la semana están encendidas las luces. Mi recomendación sería que todos 
los días sea un encendido automático en toda la universidad y por supuesto, que coloquen iluminación en los lugares en donde no hay".

No obstante anotan que todavía hay zonas en donde es deficiente la iluminación, principalmente en los alrededores del Campus. Las y los participantes señalan que:

"Se debe andar con bastante cuidado por el parqueo de Ingeniería, ya que se ve muy poco en las noches".

De igual modo, expresan que las aceras y recorridos dentro del campus universitario son peligrosos, se da la presencia de muchos huecos y aceras en mal estado lo que dificulta el transitar seguro por dichos espacios. Al respecto comentan:

"La acera entre Geología e Informática está toda quebrada".

"Hay gradas muy altas que no tienen barandas".

En cuanto a los ascensores señalan que, si bien algunos no se pueden utilizar, ya que se necesita una llave, otros son utilizados preferentemente por el profesorado y finalmente opinan que se encuentran en mal estado y tardan mucho tiempo en ser reparados. Además en cuanto a situaciones de emergencia comentan:

"Evaluar medidas de emergencia, ya que en mi caso yo utilizo silla de ruedas y en ocasiones no sirve el ascensor. Por ejemplo, ¿Qué sucede si hay un terremoto?, ¿Cómo salgo de un lugar específico?"

En cuanto a los baños señalaron que hay una tendencia a construirlos con diseño accesible, hecho que ven como algo positivo, aún así indican que las puertas son muy pesadas y eso dificulta su manipulación.

Con respecto a la accesibilidad física de otros espacios de la Sede, las y los participantes manifiestan:

"El equipo electrónico que hay para chequear en las entradas de las bibliotecas, debido a mi condición uso marcapasos y desfibrilador, no puedo permanecer durante mucho tiempo en las entradas principales. Actualmente me han dado la facilidad de ingresar por audiovisuales, sin embargo, en ocasiones debo esperar de 15 a 20 minutos para que me lleguen a atender o las puertas están cerradas, entonces hay días que no tengo tiempo de esperar, entonces no voy o envío a algún compañero. Tampoco puedo 
ingresar a Registro o a la Vicerrectoría de Acción Social, ya que están muy cargados de equipo electrónico y me provoca una sobre estimulación, entonces siempre debo de asistir acompañado y agradezco los apoyos, sin embargo, yo me siento capaz de realizar estos trámites por mi cuenta, lo que sucede es que el espacio no es adecuado". Con respecto a la ventilación indican: "Las bibliotecas están muy mal ventiladas, a mí se me limita la respiración".

En cuanto a los edificios consideran que son accesibles los siguientes: Educación, Ciencias Económicas y las Bibliotecas". Por otra parte, sobre las dificultades de accesibilidad en los edificios comentan:

"Un ejemplo de inaccesibilidad sería Letras a pesar de que en el ala C cuenta con buena iluminación, el ascensor solamente sirve para un ala".

"El edificio de Generales cuenta con dificultades en cuanto a los baños (las puertas son difíciles de abrir) y las gradas, ya que el ascensor solamente es para uso administrativo".

"La parte de debajo de Odontología no es accesible, ya que son muchas gradas. El lado de arriba si es bastante amplio".

En síntesis, en cuanto a la accesibilidad al espacio físico destacan la condición deficiente de las rampas, baños, iluminación, ascensores, aceras y recorridos en los alrededores del campus universitario, además se indican que aún cuando se visualiza un esfuerzo por mejorar la iluminación en algunas áreas de la Sede, en algunos sectores las encienden muy tarde o no lo hacen.

\section{Accesibilidad a la información}

La mayor parte de las y los participantes destacan que la información y asesoría del quehacer universitario en general son adecuadas. Así mismo, expresan que se dan fallas en cuanto a la rotulación accesible, esta es muy reducida y no tiene las características que les permite ubicarse dentro del campus. Al respecto mencionan que los "anuncios de las bibliotecas en afiches no son accesibles" y recomiendan "hacer rotulaciones en alto contraste y letras grandes, con relieve o en braille, esto en edificios como Letras". "La mayoría de los rótulos de la U son principalmente para los carros, no para los que caminamos". 
En sentido amplio, manifiestan que las Bibliotecas dan mucho apoyo al estudiantado con discapacidad visual y que el escaneo del material es adecuado; pero que aún faltan muchos recursos para obtener el material digital que requieren, en el momento que lo necesitan.

Con respecto al acceso a las bases de datos de las bibliotecas indican que:

"No hay acceso a la información en las bibliotecas para las personas con discapacidad visual. En Internet se encuentra mucha información, sin embargo, no toda es accesible para una persona con discapacidad visual o baja visión".

Además recomiendan: "realizar una base de datos de todos los libros que han sido escaneados y que posean un título".

La mayoría de las y los participantes en los grupos focales coincidieron en que la presentación de rótulos, carteles, anuncios y pizarras informativas limitan el acceso, principalmente las y los estudiantes con discapacidad visual, ya que la información está impresa, por lo que recomiendan que se mejore el sistema de información de manera digital con el fin de tener a su disposición otros mecanismos y estar al tanto de datos y anuncios relevantes para estudiantado. Igualmente, sugieren brindar más capacitación en esta área, resaltan que "En el CASED nos toman en cuenta para mejorar la accesibilidad a la información y eso es bastante bueno".

Otras recomendaciones que realizan con respecto a la accesibilidad a la información son:

"Podrían utilizar el moderador para tener acceso a otro tipo de información y no solamente a la que mandan regularmente".

"Una sugerencia sería que las personas en la biblioteca y los profesores puedan llevar cursos de LESCO".

También, indican como algo positivo que "En Derecho utilizan Facebook como una herramienta de información". En este mismo sentido, manifiestan que es necesario fortalecer la comunicación entre funcionarios administrativos y Unidades Académicas, para que el estudiantado tenga información más clara de los procesos.

En relación a la accesibilidad a la WEB, las y los estudiantes señalan:

"Algunas de las páginas WEB de información de la universidad son inaccesibles". 
"Los formatos de las imágenes que la universidad manda por correo en ocasiones son inaccesibles".

"Las guías de horarios son inaccesibles".

Y recomiendan "informar sobre las normas para crear páginas de internet 0 documentos accesibles".

De acuerdo con el acceso a la información las y los participantes en los grupos focales opinaron sobre las fallas de la rotulación, la cual no está adaptada a las características de un diseño para todas y todos.

La mayoría indica que existe una gran dificultad con las notas y anuncios que se presentan desde las pizarras informativas de las diferentes Unidades Académicas, recomiendan que se utilice un sistema alternativo de información, preferentemente a través de la WEB. Finalmente, resaltan el trabajo que realiza el Sistema de Bibliotecas Accesibles (SIBDI), en la adaptación de material impreso.

\section{Accesibilidad al proceso educativo}

Las y los participantes destacan que un aspecto positivo con respecto a la accesibilidad al proceso educativo, es la realización de los equipos de apoyo ejecutados con la coordinación del CASED, indican que "las reuniones de equipo son bastante enriquecedoras y facilitan el proceso al estudiante".

A pesar de ello, consideran que se requiere:

- Mayor capacitación a las y los docentes en cuanto a las distintas formas de aprender y de enseñar,

- mayor divulgación de servicios, principalmente acerca del apoyo y el trabajo que se lleva a cabo desde el CASED, por lo que consideran que se refuerce este aspecto.

- "EI CASED debería de abrirse un poco más, por ejemplo, en las ferias vocacionales".

A modo de recapitulación, las y los participantes en los grupos focales, perciben falta de disposición de profesores para atender a las necesidades de las y los estudiantes:

"Para que el proceso educativo sea accesible uno tiene que hacer mucho esfuerzo, porque no es inherente, ni automático (las adecuaciones), explicarle al profesor es cansado".

"Uno se gana enemigos cuando pide una adecuación”. 
"Los docentes en ocasiones se atienen a que hay estudiaderos, entonces no se preocupan mucho por brindar apoyo".

A pesar de las dificultades que han experimentado algunas y algunos estudiantes, ellos son conscientes de que: "la adecuación es el derecho mío y el deber de él (el profesor)", y anhelan una mayor empatía por parte del personal docente: "uno quisiera que se pongan en los zapatos de uno".

Las y los estudiantes con discapacidad visual manifiestan:

"No sé si avisarle a un profesor que va a tener un estudiante con discapacidad visual es lo mejor, porque es un arma de doble filo, así como lo pueden ayudar, puede estar indispuesto desde el comienzo".

"Para una persona con discapacidad visual no es viable un examen a libro abierto".

"Los pupitres siempre están desordenados, es complicado ingresar para una persona con discapacidad visual".

Además, sugieren que es necesario que las y los docentes brinden material impreso con una mejor calidad en las impresiones, tanto en la nitidez como en el tamaño y tipo de letra, máxime en los exámenes.

Otras dificultades que expresan con respecto a las adecuaciones asignadas son:

"El docente a veces olvida realizar las adecuaciones de mis exámenes y debo esperar casi media hora para que me traiga el examen, esto genera ansiedad y además mis compañeros (as) me preguntan acerca de mi discapacidad, algunos me dicen que por qué yo no uso lentes, eso es incómodo".

"En mi caso tengo que invertir mucho tiempo transcribiendo las clases que son grabadas".

En su mayoría las y los participantes de los grupos focales, expresan que se da la presencia de una actitud negativa en el profesorado, que los afecta en su proceso educativo:

"Al final del día uno se siente cansado, como una basura".

"A veces mi interés no es sacar buenas notas sino pasar el curso porque hay profesores que no lo hacen accesible, estoy en desventaja con respecto a mis compañeros". 
"Hay un estigma sobre las personas con discapacidad, son tontas, vagos, no entienden. Además los docentes actúan la mayoría del tiempo cuando la discapacidad es muy evidente".

"Los profesores piensan que uno tiene que tener una discapacidad como ser completamente sordo, ciego o andar en silla de ruedas, y no se dan cuenta que existen otro tipo de situaciones que generan una discapacidad".

"Una vez un asistente de laboratorio de biología, un muchacho de cuarto año de medicina me dijo que yo parecía una muñeca chocha, que nada me servía".

No obstante, resaltan que hay profesores con una mejor disposición para atender y responder a las necesidades propias de las lecciones, pero que presentan resistencia y actitudes negativas que ellos mismos tienen que ir "convenciéndolos" para que se dé un cambio:

"El proceso educativo se hace accesible cuando existe la disponibilidad del docente".

"Hay diversos tipos de docentes, unos colaboran bastante y otros no".

"Como estudiantes debemos aportarle al docente cuál es la necesidad que estoy presentando en un momento específico. La actitud de ambas partes es un punto vital, incluyendo al grupo de compañeros".

Para finalizar, destacan la importancia de brindar capacitación al personal docente respecto a las adecuaciones y apoyos en el proceso educativo:

"Un profesor me dijo: vea yo le puedo ayudar en todo lo que sea pero no sé como hacerlo. Me parece que capacitación a los profesores se necesita".

"Es complicado porque uno asume ser docente de los docentes para que aprendan como responder a las necesidades educativas, eso es muy pesado".

"La universidad en general tiene grandes deficiencias, los docentes no saben cómo presentar las clases".

"Hay muchos profesores que si tienen la paciencia y son muy buenos, pero también hay otros que no cooperan, se debería de sensibilizar más, capacitar a cada docente".

Las recomendaciones que expresan son las siguientes:

"El docente consejero podría apoyar un poco más los procesos del estudiante con discapacidad". 
"Los docentes deberían poder acceder a cursos del DEDUN, que les brinden estrategias didácticas".

"La Universidad podría brindar cursos de atención a la diversidad, en conjunto con la Escuela de Educación Especial".

En cuanto al acceso al proceso educativo advierten que el trabajo de los equipos de apoyo que realiza CASED, es muy positivo para la aplicación de las adecuaciones en el aula, pero que se requiere más capacitación al personal docente, así como mayor divulgación de los servicios que brinda.

La mayoría menciona como algo positivo el apoyo de algunos docentes en el proceso educativo, aún así, comentan la falta de información de que carecen las y los profesores en relación con las adecuaciones y la poca conciencia sobre este aspecto, lo cual se evidencia en las dificultades de la aplicación de las adecuaciones y apoyos del estudiante.

En síntesis, se debe resaltar que cuando se explora la accesibilidad al proceso educativo y sus experiencias, las y los estudiantes externan vivencias dolorosas, vulnerabilidad y humillaciones que inciden directamente en su desempeño académico y en su desarrollo personal social, durante su permanencia en la universidad.

En el intercambio de las opiniones durante la realización de los grupos focales, se hace evidente que parte del personal docente minimiza valores fundamentales que se deben vincular con el quehacer en el aula, como son la equidad, la solidaridad y aspectos de los derechos fundamentales de las y los estudiantes. Expresan un sentido de desprotección e imposibilidad de que la situación sea diferente y una incertidumbre que deben resolver individualmente, ya que estas experiencias no se comunican y si lo hacen no se evidencia el involucramiento de autoridades universitarias.

\section{Conclusiones}

Los grupos focales constituyen una técnica relevante de recolección de información en los procesos de investigación cualitativa, ya que establece un acercamiento personal con las y los participantes de ese proceso.

En la investigación sobre la accesibilidad en el entorno universitario los grupos focales nos permitieron conocer la percepción, opiniones, comentarios y vivencias de la vida estudiantil. De esta forma, en las dinámicas presentadas, se expresaron diferentes percepciones de las y los estudiantes en cuanto a situaciones y aspectos que les han 
permitido avanzar en su vida universitaria, así como aquellas barreras y limitaciones en el contexto de su proyecto educativo.

Es importante mencionar que cuando se utilice la técnica de grupo focal, para permitir un desarrollo empático y fluido, se consideren las características de este: número y tamaño de los de grupos, heterogeneidad de las y los participantes, así como los roles de las personas investigadoras.

En el estudio realizado, las opiniones y comentarios de la mayoría de los y las participantes de los grupos focales, coincidieron en que aún cuando los cambios que se van generando son muy reducidos y aislados (ya que el proceso administrativo y burocrático no permite que se den las transformaciones requeridas por dicha población en el momento requerido), la Universidad de Costa Rica ha hecho esfuerzos significativos por ir dando respuesta a la población con necesidades educativas asociadas o no a discapacidad,

\section{Referencias}

Boff, Leonardo y Muraro, Rose Marie. (2002). Femenino y masculino. Una nueva conciencia para el encuentro de las diferencias. Madrid, España: Trotta Ediciones.

Costa Rica, Asamblea Legislativa. (2010). Ley 8661: Convención sobre los Derechos de las personas con Discapacidad y su protocolo facultativo. San José, Costa Rica: Litografía e Imprenta LIL.

Huerta, José Manuel. (2014). Los grupos focales. Recuperado de: http://academic.uprm.edu/ihuerta/HTMLobi-94/Grupo Focal.pdf

Lema, Lorena y Agila, Liz. (2008). La lateralidad en niños y niñas en primer año de educación básica del centro educativo: San Martín de la ciudad de Cuenca período lectivo 2007-2008. (Tesis para optar por el grado de Licenciatura en Ciencias de la Educación), Universidad Politécnica Salesiana, Ecuador.

Sandoval, Carlos. (2002). Investigación Cualitativa. Bogotá, Colombia: ARFO Editores e Impresores Ltda.

Stupp, Roxana. (2005). Integración de Personas con Discapacidad a las Instituciones de Educación Superior en Costa Rica. Costa Rica: UNESCO.

Universidad de Costa Rica, Vida Estudiantil. (2013). Reglamento General de la Vicerrectoría de Vida Estudiantil. Recuperado de http://www.cu.ucr.ac.cr/normativ/vicerrectoría vida estudiantil.pdf

Vargas, Luz María. (2013). Sobre el concepto de percepción. Recuperado de http://www.redalyc.org/pdf/747/74711353004.pdf 


\section{Anexo \\ Estudiantes adscritos}

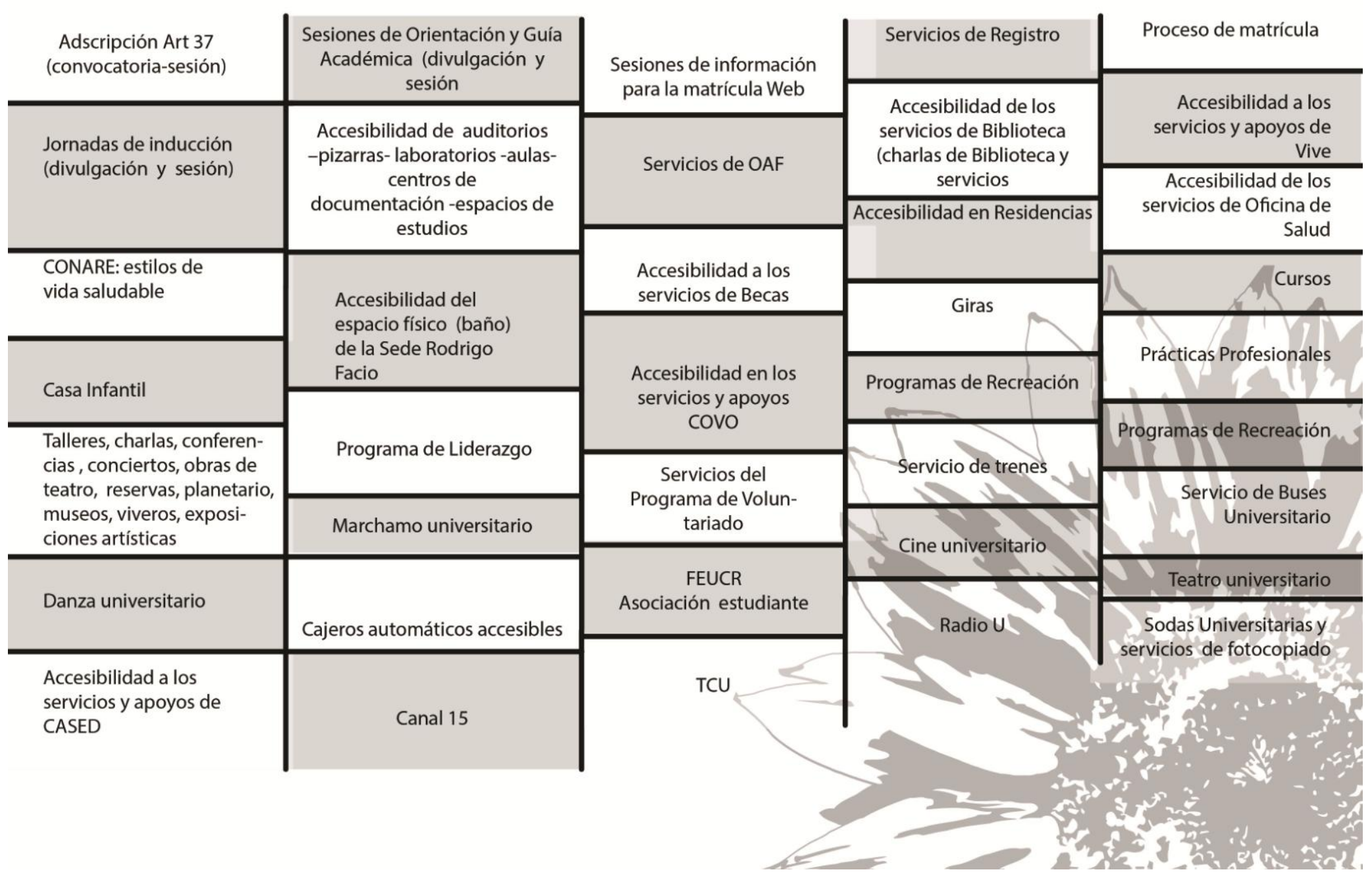

